\title{
Association of TERT and DSP variants with microscopic polyangiitis and myeloperoxidase-ANCA positive vasculitis in a Japanese population: a genetic association study
}

Aya Kawasaki ${ }^{1,2^{*}}$, Natsumi Namba ${ }^{1,2}$, Ken-ei Sada $^{3,4}$, Fumio Hirano ${ }^{5,6}$, Shigeto Kobayashi ${ }^{7}$, Kenji Nagasaka ${ }^{8}$, Takahiko Sugihara ${ }^{5,6}$, Nobuyuki Ono ${ }^{9}$, Takashi Fujimoto ${ }^{10}$, Makio Kusaoi ${ }^{11}$, Naoto Tamura ${ }^{11}$, Kunihiro Yamagata ${ }^{12}$, Takayuki Sumida $^{13}$, Hiroshi Hashimoto ${ }^{14}$, Shoichi Ozaki ${ }^{15}$, Hirofumi Makino ${ }^{16}$, Yoshihiro Arimura ${ }^{17,18}$, Masayoshi Harigai ${ }^{19}$ and Naoyuki Tsuchiya ${ }^{1,2^{*}}$ (D)

\begin{abstract}
Background: Interstitial lung disease (ILD) is a severe complication with poor prognosis in anti-neutrophil cytoplasmic antibody (ANCA)-associated vasculitis (AAV). Prevalence of AAV-associated ILD (AAV-ILD) in Japan is considerably higher than that in Europe. Recently, we reported that a MUC5B variant rs35705950, the strongest susceptibility variant to idiopathic pulmonary fibrosis (IPF), was strikingly increased in AAV-ILD patients but not in AAV patients without ILD; however, due to the low allele frequency in the Japanese population, the MUC5B variant alone cannot account for the high prevalence of AAV-ILD in Japan. In this study, we examined whether other IPF susceptibility alleles in TERT and DSP genes are associated with susceptibility to AAV subsets and AAV-ILD.
\end{abstract}

Methods: Five hundred and forty-four Japanese patients with AAV and 5558 controls were analyzed. Among the AAV patients, 432 were positive for myeloperoxidase (MPO)-ANCA (MPO-AAV). A total of 176 MPO-AAV patients were positive and 216 were negative for ILD based on CT or high-resolution CT. Genotypes of TERT and DSP variants were determined by TaqMan SNP Genotyping Assay, and their association was tested by chi-square test.

Results: When the frequencies of the IPF risk alleles TERT rs2736100A and DSP rs2076295G were compared between AAV subsets and healthy controls, both alleles were significantly increased in microscopic polyangiitis (MPA) (TERT $P=$ $2.3 \times 10^{-4}, P_{\mathrm{c}}=0.0023$, odds ratio [OR] 1.38; DSP $\left.P=6.9 \times 10^{-4}, P_{\mathrm{c}}=0.0069, \mathrm{OR} 1.32\right)$ and MPO-AAV (TERT $P=1.5 \times 10^{-4}$, $\left.P_{\mathrm{C}}=0.0015, \mathrm{OR} 1.33 ; \mathrm{DSP} P=0.0011, P_{\mathrm{C}}=0.011, \mathrm{OR} 1.26\right)$. On the other hand, no significant association was detected when the allele frequencies were compared between MPO-AAV patients with and without ILD.

(Continued on next page)

\footnotetext{
*Correspondence: a-kawasaki@umin.net; tsuchiya-tky@umin.net

${ }^{1}$ Molecular and Genetic Epidemiology Laboratory, Faculty of Medicine,

University of Tsukuba, 1-1-1 Tennodai, Tsukuba 305-8575, Japan

Full list of author information is available at the end of the article
}

\section{$\triangle B M C$}

(c) The Author(s). 2020 Open Access This article is licensed under a Creative Commons Attribution 4.0 International License, which permits use, sharing, adaptation, distribution and reproduction in any medium or format, as long as you give appropriate credit to the original author(s) and the source, provide a link to the Creative Commons licence, and indicate if changes were made. The images or other third party material in this article are included in the article's Creative Commons licence, unless indicated otherwise in a credit line to the material. If material is not included in the article's Creative Commons licence and your intended use is not permitted by statutory regulation or exceeds the permitted use, you will need to obtain permission directly from the copyright holder. To view a copy of this licence, visit http://creativecommons.org/licenses/by/4.0/ The Creative Commons Public Domain Dedication waiver (http://creativecommons.org/publicdomain/zero/1.0/) applies to the data made available in this article, unless otherwise stated in a credit line to the data. 
(Continued from previous page)

Conclusions: Unexpectedly, TERT and DSP IPF risk alleles were found to be associated with MPA and MPO-AAV, regardless of the presence of ILD. These findings suggest that TERT and DSP may be novel susceptibility genes to MPA/ MPO-AAV and also that some susceptibility genes may be shared between IPF and MPA/MPO-AAV.

Keywords: Myeloperoxidase-ANCA, Vasculitis, Microscopic polyangiitis, Susceptibility, Single nucleotide variant, Polymorphism

\section{Background}

Anti-neutrophil cytoplasmic antibody (ANCA)-associated vasculitis (AAV) is a systemic autoimmune disease characterized by ANCA production and inflammation in small vessels [1]. There are substantial differences in the epidemiology of AAV between European and Asian populations. Microscopic polyangiitis (MPA) and myeloperoxidase (MPO)-ANCA positive AAV (MPO-AAV) are predominant in East Asian populations, while granulomatosis with polyangiitis (GPA) and proteinase 3 (PR3)ANCA positive AAV (PR3-AAV) are common in the populations of European ancestry [2]. Another striking difference is that the prevalence of AAV-associated interstitial lung disease (AAV-ILD), a complication associated with poor prognosis, is considerably higher in Japanese than in European populations [3]. Among the AAV subsets, ILD was predominantly observed in MPA and MPO-AAV as compared with GPA and PR3-AAV [3]; however, significantly higher complication rate of ILD in the Japanese than in the European population is also observed when only MPA patients were compared [4], suggesting that genetic factors may contribute to the susceptibility to AAV-ILD.

Genome-wide association studies (GWAS) on AAV have been reported in European populations, and associations of HLA-DP, SERPINA1, PRTN3, and PTPN22 with GPA and PR3-AAV; HLA-DQ and PTPN22 with MPA and MPO-AAV; and HLA-DQ, BCL2L11, and TSLP with eosinophilic granulomatosis with polyangiitis (EGPA) were identified [5-8]. In a Japanese population, we reported that $H L A-D R B 1 * 09: 01-D Q B 1 * 03: 03$ haplotype and $D R B 1 * 13: 02$ were associated with risk and protection for MPA/MPO-AAV, respectively $[9,10]$. However, genetic factors of AAV have not been fully determined.

Little is known on the genetic factors associated with the occurrence of ILD among the patients with autoimmune rheumatic diseases. Recently, we reported that a single nucleotide variant (SNV) rs35705950 in the upstream region of $M U C 5 B$ gene, the strongest susceptibility variant to idiopathic pulmonary fibrosis (IPF) [11-13], was associated with ILD in the patients with rheumatoid arthritis (RA) in a multinational collaborative study [14]. Subsequently, we also reported association of rs35705950 with AAV-ILD [15]. Based on the histological and radiographic patterns of idiopathic interstitial pneumonia (IIP), ILD in AAV and RA is most frequently classified into usual interstitial pneumonia (UIP), typically observed in IPF [3]. These findings suggest a possibility that there may be shared pathological processes between IPF and AAVILD.

Although the association between $M U C 5 B$ rs35705950 and AAV-ILD is striking (odds ratio [OR] 11.6 when compared with AAV patients without ILD) [15], this allele alone cannot account for the high complication rate of ILD in Japanese AAV, because the population frequency of the risk allele is substantially lower as compared with European populations [14, 15]. Thus, other genetic factors are likely to play a role in the occurrence of ILD among AAV patients in Japan.

In addition to $M U C 5 B, T E R T$ and DSP have been reported to be associated with IPF and IIPs in GWAS $[11-13,16]$. TERT gene encodes telomerase reverse transcriptase, which is the catalytic subunit of telomerase, and contributes to maintenance of telomere length [17]. Desmoplakin, encoded by DSP, is one of desmosomal components and has a role in cell-cell adhesion and tissue integrity [18]. Although the mechanisms by which these susceptibility genes contribute to development of IPF remain unclear, TERT and DSP could be considered candidate genes which might be associated with ILD among the patients with AAV, in a similar manner to $M U C 5 B$.

This study was carried out to examine whether the IPF risk SNVs in TERT and DSP genes are associated with AAV subsets and presence of ILD among AAV patients in a Japanese population. Unexpectedly, TERT and $D S P$ SNVs turned out to be significantly associated with susceptibility to MPA and MPO-AAV regardless of the presence of ILD.

\section{Methods}

\section{Patients and controls}

Five hundred and forty-four patients with AAV and 785 healthy controls $(\mathrm{HC})$ were analyzed in this study. All patients and controls are unrelated Japanese. The patients were recruited at the institutes participating in Japan Research Committee of the Ministry of Health, Labour, and Welfare for Intractable Vasculitis (JPVAS) and Research Committee of Progressive Renal Disease, both organized by the Ministry of Health, Labour, and Welfare of Japan, and research groups organized by Tokyo Medical and Dental University and University of 
Tsukuba. The criteria for enrollment were (1) the diagnosis of AAV by site investigators, and (2) fulfilling the entry and exclusion criteria (no other diagnosis to account for symptom/signs, including malignancy, infection, drugs, and secondary vasculitis) for primary systemic vasculitis as proposed by the European Medicines Agency (EMA) algorithm [19]. The patients include those who participated in the two cohort studies previously reported by JPVAS [20, 21]. The AAV patients were classified into $315 \mathrm{MPA}, 119$ GPA, 73 EGPA, and 37 unclassifiable according to the EMA algorithm [19]. A total of 432 patients were tested positive for MPO-ANCA and 67 for PR3-ANCA by enzyme-linked immunosorbent assay (Table 1). Among the MPO-AAV patients, 176 were positive (hereafter referred to as MPO-AAV-ILD) and 216 were negative for ILD (MPO-AAV-noILD). Diagnosis of ILD was made by site investigators based on computed tomography $(\mathrm{CT})$ or high-resolution $\mathrm{CT}$ (HRCT).

Among the 785 HC, 266 samples were purchased from the Health Science Research Resources Bank (Osaka, Japan). In addition, allele frequency data of the TERT and DSP variants in 4773 individuals (4.7KJPN) was obtained from the Japanese Multi Omics Reference Panel (jMorp) [22, 23]. Together, data in 5558 individuals was used as the control data.

\section{Ethics statement}

This study was reviewed and approved by the Ethics Committees of University of Tsukuba, Tokyo Women's Medical University, Tokyo Medical and Dental University, and all other institutes participating in this study, and was conducted in accordance with the principles of the
Declaration of Helsinki. Informed consent was obtained from all subjects.

\section{Genotyping}

Genotypes of TERT rs2736100 (Assay ID: C__ 1844009_ 10) and DSP rs2076295 (Assay ID: C_16167921_10) were determined by TaqMan SNP Genotyping Assay using 7300 Real-Time PCR System (Thermo Fisher Scientific, Waltham, MA, USA).

\section{Statistical analysis}

Association was tested by chi-square test using two-by-two contingency table. $P$ values were corrected for multiple comparison (10 comparisons) by Bonferroni correction. $P$ values, corrected $P$ values $\left(P_{\mathrm{c}}\right)$, odds ratios (ORs), and 95\% confidence intervals (CIs) were calculated using $\mathrm{R}$ software (version 3.5.2). Power calculation was conducted using PS: Power and Sample Size Calculation version 3.1.6 (http:// biostat.mc.vanderbilt.edu/wiki/Main/PowerSampleSize). To calculate the power, the significance level was set at 0.05 . The power to detect association with $\mathrm{OR}=1.2,1.4,1.6$, and 1.8 is shown in Additional file 1: Supplementary Table S1.

\section{Results \\ Association of TERT and DSP IPF risk SNVs with MPA and MPO-AAV}

Genotypes for TERT rs2736100 and DSP rs2076295 were determined in 544 patients with AAV and $785 \mathrm{HC}$. No deviation from the Hardy-Weinberg equilibrium was observed (rs2736100, AAV: $P=0.25$, HC: $P=0.89$; rs2076295, AAV: $P=0.27$, HC: $P=0.91$ ).

Initially, we examined whether TERT and DSP are associated with susceptibility to AAV subsets. AAV patients

Table 1 Characteristics in AAV patients and controls

\begin{tabular}{llll}
\hline Characteristics & Groups & Number & Female/male \\
\hline AAV & & & \\
EMA classification & MPA & 315 & $192 / 123$ \\
& GPA & 73 & $47 / 52$ \\
& EGPA & $73 / 27$ \\
ANCA specificity & Unclassifiable & 37 & $24 / 13$ \\
& MPO-AAV & 432 & $268 / 164$ \\
Presence/absence of ILD in MPO-AAV & PR3-AAV & 67 & $33 / 34$ \\
& MPO-AAV-ILD & 176 & $101 / 75$ \\
Controls & MPO-AAV-nolLD & 216 & $139 / 77$ \\
& HC & 785 & $478 / 307$ \\
\hline A & $4.7 K J P N^{c}$ & 4773 & $2641 / 2130$ \\
\hline
\end{tabular}

AAV ANCA-associated vasculitis, EMA European Medicines Agency, MPA microscopic polyangiitis, GPA granulomatosis with polyangiitis, EGPA eosinophilic granulomatosis with polyangiitis, MPO-AAV myeloperoxidase (MPO)-ANCA positive AAV, PR3-AAV proteinase 3 (PR3)-ANCA positive AAV, ILD interstitial lung disease, MPO-AAV-ILD MPO-AAV with ILD, MPO-AAV-noILD MPO-AAV without ILD

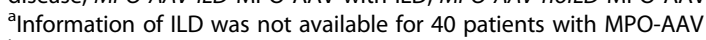

${ }^{b}$ Healthy controls recruited by our research group

${ }^{\mathrm{c}}$ Allele frequency data in 4.7KJPN was obtained from the Japanese Multi Omics Reference Panel (jMorp) $[22,23]$ 
were classified according to the EMA algorithm (MPA, GPA, and EGPA) [19], or ANCA specificity (MPO-AAV and PR3-AAV). TERT rs2736100A was significantly increased in MPA $\left(P=2.3 \times 10^{-4}, P_{\mathrm{c}}=0.0023\right.$, OR $1.38,95 \%$ CI 1.16-1.64) and MPO-AAV $\left(P=1.5 \times 10^{-4}, P_{\mathrm{c}}=0.0015\right.$, OR 1.33, 95\% CI 1.15-1.54) when compared with controls (Table 2). Similarly, DSP rs2076295G was significantly increased in MPA $\left(P=6.9 \times 10^{-4}, P_{\mathrm{c}}=0.0069\right.$, OR $1.32,95 \%$ CI 1.12-1.55) and MPO-AAV $\left(P=0.0011, P_{\mathrm{c}}=0.011\right.$, OR $1.26,95 \%$ CI 1.10-1.45) (Table 2). A tendency towards association of TERT rs2736100A with EGPA and PR3-AAV, and that of DSP rs2076295G with PR3-AAV was observed, although the association did not reach statistical significance after Bonferroni correction for multiple testing.

To exclude the possible effect caused by the difference in female-to-male ratio between AAV and controls, female and male subjects were separately tested for association. As shown in Table 3, the same trend towards association of TERT rs2736100A was observed both in the female and male subjects. On the other hand, the association of DSP rs2076295G was detected in male (MPA: $P=2.3 \times 10^{-5}$, OR $1.76,95 \%$ CI $1.35-2.29$; MPOAAV: $P=3.5 \times 10^{-6}$, OR $1.71,95 \%$ CI $\left.1.36-2.15\right)$, but not in female individuals.

\section{Lack of association of TERT and DSP IPF risk SNVs with presence of ILD in MPO-AAV}

We next investigated whether the TERT rs2736100A and DSP rs2076295G contribute to the development of ILD in AAV. Because the prevalence of ILD is higher in MPO-AAV compared with PR3-AAV [3], and the TERT and DSP SNVs were associated with susceptibility to
MPO-AAV as described above, only MPO-AAV patients were examined for the association of these SNVs with the presence of ILD in a case-case study comparing MPO-AAV patients with ILD (MPO-AAV-ILD) and those without ILD (MPO-AAV-noILD) (Table 4). No significant association was detected in TERT rs2736100 $(P=0.37$, OR $0.87,95 \%$ CI $0.65-1.18)$ nor in DSP rs2076295 ( $P=0.52$, OR $1.10,95 \%$ CI $0.83-1.46)$.

\section{Discussion}

Here, we report that TERT rs2736100A and DSP rs2076295G, both of which are the risk alleles for IPF $[12,13,16]$, are associated with susceptibility to MPA and MPO-AAV in a Japanese population. Unexpectedly, association of these alleles with occurrence of ILD among the patients with MPO-AAV was not detected. These are new findings which have not been reported in the populations of European nor of Asian ancestry.

It has been recognized that some patients with IPF are positive for MPO-ANCA and develop MPA [24], and hypothesized that IPF and AAV may share some pathogenic mechanisms. Our current finding that the IPF risk alleles of TERT and DSP are associated with MPA and MPO-AAV is in line with such hypothesis. This is also supported by the reports that silica contributes to the pathogenesis of both IPF and AAV [25, 26].

On the other hand, significant association of TERT and DSP IPF risk SNVs was not detected when MPOAAV patients with and without ILD were compared. This finding was unexpected, but similar finding has been reported in RA, where association of these genes was not observed in RA-ILD when compared with RA

Table 2 Association of TERT and DSP with MPA and MPO-AAV

\begin{tabular}{|c|c|c|c|c|c|c|c|c|}
\hline & \multicolumn{4}{|c|}{ TERT rs2736100A } & \multicolumn{4}{|c|}{ DSP rs2076295G } \\
\hline & $n$ (AF) & $P$ & $P_{\mathrm{c}}$ & OR $(95 \% \mathrm{Cl})$ & $n(\mathrm{AF})$ & $P$ & $P_{\mathrm{c}}$ & OR $(95 \% \mathrm{Cl})$ \\
\hline \multicolumn{9}{|l|}{$\overline{A A V}$} \\
\hline MPA & $427(0.678)$ & $2.3 \times 10^{-4}$ & 0.0023 & $1.38(1.16-1.64)$ & $351(0.557)$ & $6.9 \times 10^{-4}$ & 0.0069 & $1.32(1.12-1.55)$ \\
\hline GPA & $142(0.597)$ & 0.82 & 1 & $0.97(0.75-1.26)$ & $130(0.546)$ & 0.074 & 0.74 & $1.26(0.98-1.64)$ \\
\hline EGPA & $101(0.692)$ & 0.031 & 0.31 & $1.47(1.03-2.09)$ & $77(0.527)$ & 0.34 & 1 & $1.17(0.85-1.63)$ \\
\hline MPO-AAV & $577(0.669)$ & $1.5 \times 10^{-4}$ & 0.0015 & $1.33(1.15-1.54)$ & $471(0.545)$ & 0.0011 & 0.011 & $1.26(1.10-1.45)$ \\
\hline PR3-AAV & $93(0.694)$ & 0.034 & 0.34 & $1.49(1.03-2.15)$ & $78(0.582)$ & 0.03 & 0.3 & $1.46(1.04-2.07)$ \\
\hline \multicolumn{9}{|l|}{ Controls } \\
\hline$H C^{a}$ & $965(0.615)$ & & & & $791(0.505)$ & & & \\
\hline 4.7KJPN ${ }^{b}$ & $5747(0.602)$ & & & & $4628(0.485)$ & & & \\
\hline All controls & $6712(0.604)$ & & & Referent & $5419(0.488)$ & & & Referent \\
\hline \multicolumn{9}{|c|}{$\begin{array}{l}P \text { values were calculated by chi-square test in comparison between each AAV subset and all controls. Correction for multiple testing was done by Bonferroni } \\
\text { correction. Corrected } P \text { values }\left(P_{\mathrm{c}}\right) \text { were calculated by multiplying uncorrected } P \text { values by } 10 \text {. Significant association after correction for multiple testing }\left(P_{\mathrm{c}}<\right. \\
\text { is shown in bold } \\
A A V \text { ANCA-associated vasculitis, } n \text { allele count, } A F \text { allele frequency, MPA microscopic polyangitis, GPA granulomatosis with polyangiitis, EGPA eosinophilic } \\
\text { granulomatosis with polyangiitis, MPO-AAV myeloperoxidase (MPO)-ANCA positive vasculitis, PR3-AAV proteinase } 3 \text { (PR3)-ANCA positive vasculitis, HC healthy } \\
\text { controls, } P_{c} \text { corrected } P \text { values, OR odds ratio, } 95 \% C I 95 \% \text { confidence interval } \\
\text { a Healthy controls recruited by our research group }\end{array}$} \\
\hline
\end{tabular}


Table 3 Association of TERT and DPS with AAV in female and male individuals

\begin{tabular}{|c|c|c|c|c|c|c|}
\hline & \multicolumn{3}{|c|}{ TERT rs2736100A } & \multicolumn{3}{|c|}{ DSP rs2076295G } \\
\hline & $n(\mathrm{AF})$ & $P$ & OR $(95 \% \mathrm{Cl})$ & $\overline{n(\mathrm{AF})}$ & $P$ & OR $(95 \% \mathrm{Cl})$ \\
\hline \multicolumn{7}{|l|}{ Female } \\
\hline MPA & $260(0.677)$ & 0.0014 & $1.43(1.15-1.78)$ & $198(0.516)$ & 0.35 & $1.10(0.90-1.36)$ \\
\hline MPO-AAV & $358(0.670)$ & $6.0 \times 10^{-4}$ & $1.39(1.15-1.67)$ & $269(0.502)$ & 0.62 & $1.05(0.88-1.25)$ \\
\hline Controls ${ }^{a}$ & $3708(0.595)$ & & Referent & $3062(0.491)$ & & Referent \\
\hline \multicolumn{7}{|l|}{ Male } \\
\hline MPA & $167(0.679)$ & 0.049 & $1.32(1.00-1.73)$ & $153(0.622)$ & $2.3 \times 10^{-5}$ & $1.76(1.35-2.29)$ \\
\hline MPO-AAV & $219(0.668)$ & 0.064 & $1.25(0.99-1.58)$ & $202(0.616)$ & $3.5 \times 10^{-6}$ & $1.71(1.36-2.15)$ \\
\hline Controls ${ }^{a}$ & $3003(0.616)$ & & Referent & $2355(0.484)$ & & Referent \\
\hline
\end{tabular}

$P$ values were calculated by chi-square test in comparison between each AAV subset and controls AAV ANCA-associated vasculitis, MPA microscopic polyangiitis, MPO-AAV myeloperoxidase (MPO)-ANCA positive vasculitis, $n$ allele count, $A F$ allele frequency, OR odds ratio, 95\% Cl 95\% confidence interval

a Data in controls include allele data in healthy controls and 4.7KJPN obtained from the Japanese Multi Omics Reference Panel (jMorp) [22, 23]

patients without ILD [14]. This is in contrast to $M U C 5 B$, which shows association in the patients with AAV and RA only when they are complicated by ILD [14, 15]. These results might suggest a possibility that some IPF associated genes (e.g., $M U C 5 B)$ may play a role in the process of lung disease, and others (e.g., TERT and DSP) may be involved in the shared molecular background between AAV/RA and IPF. Further studies are required to validate this hypothesis.

The association of TERT rs2736100 was reported in GWAS on IPF in a Japanese population [16], and also in GWAS on IIPs, among which IPF was the most common subset, in European populations [12]. TERT rs2736100 is located in intron 2 of the TERT gene. Wei et al. reported that the TERT risk allele, rs2736100A, showed lower enhancer activity compared with rs $2736100 \mathrm{C}$, using a luciferase assay in primary lung epithelial cells [27]. They also found that rs2736100A showed decreased expression of TERT mRNA [27], suggesting a functional significance of this SNV. Moreover, rs2736100A was associated with shorter leukocyte telomere length [28], previously shown to contribute to risk of IPF [29]. With respect to AAV, a proportion of $\mathrm{T}$ cells was reported to show short telomeres in GPA patients [30], although telomere length abnormality has not been reported in MPA or MPO-AAV. Because TERT has an anti-apoptotic effect [31], decreased expression of TERT associated with the risk allele may result in an enhancement of apoptosis. Indeed, in AAV patients, an enhanced rate of apoptosis was observed in neutrophils [32]. Apoptotic neutrophils may be opsonized with anti-MPO and anti-PR3 antibodies which recognize MPO and PR3 on the surface, engulfed by macrophages, and lead to secretion of pro-inflammatory cytokines such as interleukin-1 and interleukin-8. These processes may induce inflammation in vessel and tissue injury [33, 34].

Interestingly, association of TERT rs7726159A has been shown to be associated with systemic lupus erythematosus (SLE) in East Asian populations [35]. The risk SNV for SLE is in moderate linkage disequilibrium $\left(r^{2}=\right.$ 0.773) with that for MPO-AAV and IPF, although the risk allele is the opposite. Nevertheless, this finding may suggest that TERT variants may be functional and associated with multiple autoimmune conditions either in a predispositional or in a protective manner, regardless of the presence of ILD.

The association of DSP with IIPs and IPF has been identified by GWAS in the European populations [12, 13]. DSP rs2076295 is located in intron 5, and the risk allele was reported to be associated with decreased expression of DSP in the lung [12]. Expression quantitative trait locus (eQTL) analysis using the GTEx Portal database [36] shows that rs2076295G is the most strongly associated variant with DSP expression in the lung $\left(P=3.7 \times 10^{-75}\right.$, normalized effect size $=-0.73$, Additional file 2: Figure S1).

DSP was reported to modulate Wnt/beta-catenin signaling, which is involved in cell proliferation, differentiation,

Table 4 Association study of TERT and DSP in MPO-AAV with ILD

\begin{tabular}{|c|c|c|c|c|c|c|}
\hline & \multicolumn{3}{|c|}{ TERT rs2736100A } & \multicolumn{3}{|c|}{ DSP rs2076295G } \\
\hline & $n$ (AF) & $P$ & OR (95\% Cl) & $n$ (AF) & $P$ & OR $(95 \% \mathrm{Cl})$ \\
\hline MPO-AAV-ILD & $230(0.653)$ & 0.37 & $0.87(0.65-1.18)$ & $198(0.563)$ & 0.52 & $1.10(0.83-1.46)$ \\
\hline MPO-AAV-nolLD & $294(0.684)$ & & Referent & $233(0.539)$ & & Referent \\
\hline
\end{tabular}

$P$ values were calculated by chi-square test in comparison between MPO-AAV-ILD and MPO-AAV-nolLD. MPO-AAV myeloperoxidase-ANCA positive vasculitis, ILD interstitial lung disease, MPO-AAV-ILD MPO-AAV with ILD, MPO-AAV-noILD MPO-AAV without ILD, $n$ allele count, $A F$ allele frequency, OR odds ratio, 95\% CI 95\% confidence interval 
immune responses, and carcinogenesis [37, 38]. In Dspdeficient atrial myocyte cell lines and HEK293T cells transfected with DSP frameshift variant, the Wnt/beta-catenin signaling was suppressed $[39,40]$. Although contribution of the Wnt signaling to the pathogenesis of AAV is unclear, Wnt signaling has been reported to play a role in autoimmune diseases such as SLE and RA [41]. In this study, association of DSP was observed in male but not in female AAV. Although the reason of such difference remains unclear, sex hormone or genes located in sex chromosomes might affect the association of DSP.

Our study has several limitations. Due to rarity of AAV, a replication study was not conducted. The annual incidence/million in Japan has been reported to be 22.6 and 18.2 for AAV and MPA, respectively [2]. As shown in Additional file 1: Supplementary Table S1, when OR is less than 1.4, we cannot detect the association with power $\geq 0.8$ in the case-case analysis comparing AAV patients with and without ILD. Therefore, the results in this study should be confirmed in larger sample size in the future. In addition, not all AAV patients with ILD were diagnosed by HRCT, and lung biopsy was not performed in most of the patients. Thus, we cannot exclude the possibility that ILD with a specific histological pattern such as UIP might show association with TERT or DSP IPF risk SNVs.

\section{Conclusions}

TERT and DSP IPF risk SNVs were found to be associated with susceptibility to MPA and MPO-AAV for the first time. On the other hand, significant association with complication of ILD in AAV was not detected. Our findings suggested that some susceptibility genes are shared between IPF and AAV, regardless of the presence of ILD.

\section{Supplementary information}

Supplementary information accompanies this paper at https://doi.org/10. 1186/s13075-020-02347-0.

\section{Additional file 1: Supplementary Table S1. Power to detect} associations under the sample size in this study.

Additional file 2: Supplementary Figure S1. DSP rs2076295G is associated with lower expression of DSP mRNA in lung.

\footnotetext{
Abbreviations

AAV: Anti-neutrophil cytoplasmic antibody-associated vasculitis; ANCA: Antineutrophil cytoplasmic antibody; Cl: Confidence interval; CT: Computed tomography; EGPA: Eosinophilic granulomatosis with polyangiitis; EMA: European Medicines Agency; eQTL: Expression quantitative trait locus; GPA: Granulomatosis with polyangiitis; GWAS: Genome-wide association studies; HC: Healthy controls; HRCT: High-resolution computed tomography; IIP: Idiopathic interstitial pneumonia; ILD: Interstitial lung disease; IPF: Idiopathic pulmonary fibrosis; MPA: Microscopic polyangiitis; MPO: Myeloperoxidase; OR: Odds ratio; PR3: Proteinase 3; RA: Rheumatoid arthritis; SLE: Systemic lupus erythematosus; SNV: Single nucleotide variant; UIP: Usual interstitial pneumonia
}

\section{Acknowledgements}

The authors are grateful to all the patients for participating in this study and to the clinical staff associated with Japan Research Committee of the Ministry of Health, Labour, and Welfare for Intractable Vasculitis (JPVAS) and Research Committee of Progressive Renal Disease of the Ministry of Health, Labour, and Welfare of Japan for recruiting the patients and collecting clinical information.

\section{Authors' contributions}

Drs. Kawasaki, Namba, and Tsuchiya conceived and designed the study, performed the genotyping and statistical analysis, interpreted the data, and wrote the manuscript. Drs. Sada, Hirano, Kobayashi, Nagasaka, Sugihara, Ono, Fujimoto, Kusaoi, Tamura, Yamagata, and Sumida recruited the patients and analyzed the clinical data. Drs. Ozaki, Hashimoto, Makino, Arimura, and Harigai organized the study groups, conceived the study, and interpreted the data. All authors read and approved the manuscript.

\section{Funding}

This study was supported by the Japan Agency for Medical Research and Development "Multitiered study to address clinical questions for management of intractable vasculitides [grant number 19ek0109360h002]" and research grants from Bristol-Myers Squibb, Japan College of Rheumatology, and Japan Rheumatism Association.

\section{Availability of data and materials}

The data that support the findings of this study are included in this published article and its Supplementary Information. Other data are available from the corresponding authors [NT and AK] on reasonable request. However, the genotype data and clinical information of each individual participant are not available, based on the Act on the Protection of Personal Information enforced in Japan and the conditions on which the informed consent was given.

\section{Ethics approval and consent to participate}

This study was reviewed and approved by the Ethics Committees of University of Tsukuba Faculty of Medicine (No. 180, 227, 268), Tokyo Women's Medical University, Tokyo Medical and Dental University, and all other institutes participating in this study, and was conducted in accordance with the principles of the Declaration of Helsinki. Informed consent was obtained from all participants.

\section{Consent for publication}

Not applicable

\section{Competing interests}

Dr. Sada has received speaker's honoraria from Chugai Pharmaceutical Co. Ltd.

Dr. Hirano has received research grants and/or speaker's honoraria from Chugai Pharmaceutical Co. Ltd., Ono Pharmaceuticals, CSL Behring, Towa Pharmaceutical Co. Ltd., Abbvie Japan Co. Ltd., Japan Blood Products Organization, Ayumi Pharmaceutical Co., Nippon Kayaku Co. Ltd., Astellas Pharma Inc., Sumitomo Dainippon Pharma, and UCB Japan.

Dr. Nagasaka has received speaker's honoraria from Bristol-Myers Squibb Co. Ltd., Chugai Pharmaceutical Co. Ltd., and Teijin Pharma Ltd.

Dr. Makino is a consultant for AbbVie, Teijin, and Boehringer-Ingelheim. Dr. Harigai has received research grants and/or honoraria from AbbVie Japan Co. Ltd., Bristol-Myers Squibb K.K., Chugai Pharmaceutical Co. Ltd., Eisai Co. Ltd., Mitsubishi Tanabe Pharma Co., Ono Pharmaceutical Co. Ltd., Taisho Toyama Pharmaceutical Co. Ltd., Ayumi Pharmaceutical Co. Ltd., Takeda Pharmaceutical Co. Ltd., Teijin Pharma Ltd., and Pfizer Japan Inc. Dr. Harigai serves as a consultant for Bristol-Myers Squibb K.K., Chugai Pharmaceutical Co. Ltd., CIMIC Co. Ltd., Kissei Pharmaceutical Co. Ltd., Ono Pharmaceutical Co. Ltd., and Pfizer Japan Inc.

Dr. Tsuchiya has received research fund from H.U. Group Research Institute G.K. for a collaborative research unrelated to this study, and research grants from Bristol-Myers Squibb, 2015 Japan College of Rheumatology Award from Japan College of Rheumatology, 2017 Novartis Rheumatology Award from Japan Rheumatism Association with research funding, and speaker's honoraria from Teijin.

Other authors declare no potential conflict of interest. 


\section{Author details}

Molecular and Genetic Epidemiology Laboratory, Faculty of Medicine, University of Tsukuba, 1-1-1 Tennodai, Tsukuba 305-8575, Japan. ${ }^{2}$ School of Medical Sciences, University of Tsukuba, Tsukuba, Japan. ${ }^{3}$ Department of Nephrology, Rheumatology, Endocrinology and Metabolism, Graduate School of Medicine, Dentistry and Pharmaceutical Sciences, Okayama University, Okayama, Japan. ${ }^{4}$ Department of Clinical Epidemiology, Kochi Medical School, Kochi University, Nankoku, Japan. ${ }^{5}$ Department of Rheumatology, Graduate School of Medical and Dental Sciences, Tokyo Medical and Dental University, Tokyo, Japan. ${ }^{6}$ Department of Lifetime Clinical Immunology, Graduate School of Medical and Dental Sciences, Tokyo Medical and Dental University, Tokyo, Japan. ${ }^{7}$ Department of Internal Medicine, Juntendo University Koshigaya Hospital, Koshigaya, Japan. ${ }^{8}$ Department of Rheumatology, Ome Municipal General Hospital, Ome, Japan. ${ }^{9}$ Department of Rheumatology, Saga University, Saga, Japan. ${ }^{10}$ The Center for Rheumatic Diseases, Nara Medical University, Kashihara, Japan. ${ }^{11}$ Department of Internal Medicine and Rheumatology, School of Medicine, Juntendo University, Tokyo, Japan. ${ }^{12}$ Department of Nephrology, Faculty of Medicine, University of Tsukuba, Tsukuba, Japan. ${ }^{13}$ Department of Internal Medicine, Faculty of Medicine, University of Tsukuba, Tsukuba, Japan. ${ }^{14}$ School of Medicine, Juntendo University, Tokyo, Japan. ${ }^{15}$ Department of Internal Medicine, St. Marianna University School of Medicine, Kawasaki, Japan. ${ }^{16}$ Okayama University, Okayama, Japan. ${ }^{17}$ Department of Nephrology and Rheumatology, Kyorin University School of Medicine, Mitaka, Japan. ${ }^{18}$ Department of Internal Medicine, Kichijoji Asahi Hospital, Musashino, Japan. ${ }^{19}$ Department of Rheumatology, School of Medicine, Tokyo Women's Medical University, Tokyo, Japan.

Received: 23 June 2020 Accepted: 6 October 2020

Published online: 16 October 2020

\section{References}

1. Xiao H, Hu P, Falk RJ, Jennette JC. Overview of the pathogenesis of ANCAassociated vasculitis. Kidney Dis. 2016;1:205-15

2. Fujimoto S, Watts RA, Kobayashi S, Suzuki K, Jayne DR, Scott DG, et al. Comparison of the epidemiology of anti-neutrophil cytoplasmic antibodyassociated vasculitis between Japan and the U.K. Rheumatology. 2011;50: 1916-20.

3. Alba MA, Flores-Suárez LF, Henderson AG, Xiao H, Hu P, Nachman PH, et al. Interstital lung disease in ANCA vasculitis. Autoimmun Rev. 2017;16:722-9.

4. Furuta S, Chaudhry AN, Hamano Y, Fujimoto S, Nagafuchi $H$, Makino $H$, et al. Comparison of phenotype and outcome in microscopic polyangiitis between Europe and Japan. J Rheumatol. 2014;41:325-33.

5. Lyons PA, Rayner TF, Trvedi S, Holle JU, Watts RA, Jayne DR, et al. Genetically distinct subsets within ANCA-associated vasculitis. N Engl J Med. 2012:367:214-23.

6. Xie G, Roshandel D, Sherva R, Monach PA, Lu EY, Kung T, et al. Association of granulomatosis with polyangiitis (Wegener's) with HLA-DPB1*04 and SEMA6A gene variants: evidence from genome-wide analysis. Arthritis Rheum. 2013:65:2457-68.

7. Merkel PA, Xie G, Monach PA, Ji X, Ciavatta DJ, Byun J, et al. Identification of functional and expression polymorphisms associated with risk for antineutrophil cytoplasmic autoantibody-associated vasculitis. Arthritis Rheumatol. 2017;69:1054-66.

8. Lyons PA, Peters JE, Alberici F, Liley J, Coulson RMR, Astle W, et al. Genomewide association study of eosinophilic granulomatosis with polyangiitis reveals genomic loci stratified by ANCA status. Nat Commun. 2019;10:5120

9. Tsuchiya N, Kobayashi S, Kawasaki A, Kyogoku C, Arimura Y, Yoshida M, et al. Genetic background of Japanese patients with antineutrophil cytoplasmic antibody-associated vasculitis: association of HLA-DRB1*0901 with microscopic polyangiitis. J Rheumatol. 2003;30:1534-40.

10. Kawasaki A, Hasebe N, Hidaka M, Hirano F, Sada KE, Kobayashi S, et al. Protective role of HLA-DRB1*13:02 against microscopic polyangiitis and MPO-ANCA-positive vasculitides in a Japanese population: a case-control study. PLoS One. 2016;11:e0154393.

11. Seibold MA, Wise AL, Speer MC, Steele MP, Brown KK, Loyd JE, et al. A common MUC5B promoter polymorphism and pulmonary fibrosis. N Engl J Med. 2011:364:1503-12

12. Fingerlin TE, Murphy E, Zhang W, Peljto AL, Brown KK, Steele MP, et al. Genome-wide association study identifies multiple susceptibility loci for pulmonary fibrosis. Nat Genet. 2013;45:613-20.
13. Allen RJ, Porte J, Braybrooke R, Flores C, Fingerlin TE, Oldham JM, et al. Genetic variants associated with susceptibility to idiopathic pulmonary fibrosis in people of European ancestry: a genome-wide association study. Lancet Respir Med. 2017:5:869-80.

14. Juge PA, Lee JS, Ebstein E, Furukawa H, Dobrinskikh E, Gazal S, et al. MUC5B promoter variant and rheumatoid arthritis with interstitial lung disease. N Engl J Med. 2018;379:2209-19.

15. Namba N, Kawasaki A, Sada KE, Hirano F, Kobayashi S, Yamada H, et al. Association of MUC5B promoter polymorphism with interstitial lung disease in myeloperoxidase-antineutrophil cytoplasmic antibody-associated vasculitis. Ann Rheum Dis. 2019;78:1144-6.

16. Mushiroda T, Wattanapokayakit S, Takahashi A, Nukiwa T, Kudoh S, Ogura T, et al. A genome-wide association study identifies an association of a common variant in TERT with susceptibility to idiopathic pulmonary fibrosis. Med Genet. 2008:45:654-6.

17. Nagpal N, Agarwal S. Telomerase RNA processing: implications for human health and disease. Stem Cells. 2020. doi:https://doi.org/10.1002/stem.3270.

18. Kowalczyk AP, Green KJ. Structure, function, and regulation of desmosomes. Prog Mol Biol Transl Sci. 2013;116:95-118.

19. Watts R, Lane S, Hanslik T, Hauser T, Hellmich B, Koldingsnes W, et al. Development and validation of a consensus methodology for the classification of the ANCA-associated vasculitides and polyarteritis nodosa for epidemiological studies. Ann Rheum Dis. 2007;66:222-7.

20. Sada KE, Yamamura M, Harigai M, Fujii T, Dobashi H, Takasaki Y, et al. Classification and characteristics of Japanese patients with antineutrophil cytoplasmic antibody-associated vasculitis in a nationwide, prospective, inception cohort study. Arthritis Res Ther. 2014;16:R101.

21. Sada KE, Harigai M, Amano K, Atsumi T, Fujimoto S, Yuzawa Y, et al Comparison of severity classification in Japanese patients with antineutrophil cytoplasmic antibody-associated vasculitis in a nationwide, prospective, inception cohort study. Mod Rheumatol. 2016;26:730-7.

22. Tadaka S, Saigusa D, Motoike IN, Inoue J, Aoki Y, Shirota M, et al. jMorp: Japanese Multi Omics Reference Panel. Nucleic Acids Res. 2018;46:D551-7.

23. Japanese Multi Omics Reference Panel (jMorp). https://jmorp.megabank. tohoku.ac.jp/202001. Accessed 11 Mar 2020.

24. Kagiyama N, Takayanagi N, Kanauchi T, Ishiguro T, Yanagisawa T, Sugita Y. Antineutrophil cytoplasmic antibody-positive conversion and microscopic polyangiitis development in patients with idiopathic pulmonary fibrosis. BMJ Open Respir Res. 2015;2:e000058.

25. Cohen RA, Patel A, Green FH. Lung disease caused by exposure to coal mine and silica dust. Semin Respir Crit Care Med. 2008;29:651-61.

26. Scott DGl, Watts RA. Epidemiology and clinical features of systemic vasculitis. Clin Exp Nephrol. 2013;17:607-10.

27. Wei R, Cao L, Pu H, Wang H, Zheng Y, Niu X, et al. TERT polymorphism rs $2736100-C$ is associated with EGFR mutation-positive non-small cell lung cancer. Clin Cancer Res. 2015:21:5173-80.

28. Codd V, Nelson CP, Albrecht E, Mangino M, Deelen J, Buxton JL, et al. Identification of seven loci affecting mean telomere length and their association with disease. Nat Genet. 2013;45:422-7.

29. Alder JK, Chen JJ, Lancaster L, Danoff S, Su SC, Cogan JD, et al. Short telomeres are a risk factor for idiopathic pulmonary fibrosis. Proc Natl Acad Sci U S A. 2008:105:13051-6.

30. Vogt S, Iking-Konert C, Hug F, Andrassy K, Hänsch GM. Shortening of telomeres: evidence for replicative senescence of $T$ cells derived from patients with Wegener's granulomatosis. Kidney Int. 2003;63:2144-51.

31. Sung $Y H$, Choi YS, Cheong C, Lee HW. The pleiotropy of telomerase against cell death. Mol Cells. 2005;19:303-9.

32. Harper L, Cockwell P, Adu D, Savage CO. Neutrophil priming and apoptosis in anti-neutrophil cytoplasmic autoantibody-associated vasculitis. Kidney Int. 2001;59:1729-38.

33. Schreiber A, Pham CTN, Hu Y, Schneider W, Luft FC, Kettritz R. Neutrophil serine proteases promote $\mathrm{IL}-1 \beta$ generation and injury in necrotizing crescentic glomerulonephritis. J Am Soc Nephrol. 2012;23: 470-82

34. Cartin-Ceba R, Peikert T, Specks U. Pathogenesis of ANCA-associated vasculitis. Curr Rheumatol Rep. 2012;14:481-93.

35. Sun C, Molineros JE, Looger LL, Zhou XJ, Kim K, Okada Y, et.al. High-density genotyping of immune-related loci identifies new SLE risk variants in individuals with Asian ancestry. Nat Genet 2016:48:323-330.

36. GTEx Portal. https://gtexportal.org/home. Accessed 24 Apr 2020. 
37. Pai SG, Carneiro BA, Mota JM, Costa R, Leite CA, Barroso-Sousa R, et al. Wht/ beta-catenin pathway: modulating anticancer immune response. J Hematol Oncol. 2017;10:101.

38. Chae WJ, Bothwell ALM. Canonical and non-canonical Wnt signaling in immune cells. Trends Immunol. 2018;39:830-47.

39. Garcia-Gras E, Lombardi R, Giocondo MJ, Willerson JT, Schneider MD, Khoury DS, et al. Suppression of canonical Wnt/beta-catenin signaling by nuclear plakoglobin recapitulates phenotype of arrhythmogenic right ventricular cardiomyopathy. J Clin Invest. 2006;116:2012-21.

40. Lin X, Ma Y, Cai Z, Wang Q, Wang L, Huo Z, et al. Next-generation sequencing identified novel desmoplakin frame-shift variant in patients with arrhythmogenic cardiomyopathy. BMC Cardiovasc Disord. 2020;20:74.

41. Shi J, Chi S, Xue J, Yang J, Li F, Liu X. Emerging role and therapeutic implication of Wnt signaling pathways in autoimmune diseases. J Immunol Res. 2016;2016:9392132.

\section{Publisher's Note}

Springer Nature remains neutral with regard to jurisdictional claims in published maps and institutional affiliations.

Ready to submit your research? Choose BMC and benefit from:

- fast, convenient online submission

- thorough peer review by experienced researchers in your field

- rapid publication on acceptance

- support for research data, including large and complex data types

- gold Open Access which fosters wider collaboration and increased citations

- maximum visibility for your research: over $100 \mathrm{M}$ website views per year

At BMC, research is always in progress.

Learn more biomedcentral.com/submissions 